\title{
COVID-19: frequently asked questions to the cardiologist
}

\author{
Andreas A. Kammerlander · Julia Mascherbauer
}

In December 2019, a previously unknown betacoronavirus was identified in a cluster of patients with pneumonia in Wuhan, China [1]. The pathogen was later named severe acute respiratory syndrome coronavirus 2 (SARS-CoV-2), sharing similarities with already known SARS strains. The disease caused by SARS-CoV-2 was named coronavirus disease 2019 (COVID-19) [2].

The main symptoms of COVID-19 are cough and fever, although a broad spectrum of symptoms have been described, including the absence of both fever and cough in patients admitted to the intensive care unit (ICU) with acute respiratory syndrome (ARDS) in need of mechanical ventilation [2].

Given the presumably high number of undetected mild COVID-19 cases, exact case fatality rates are currently unknown [3, 4]. Identification of patients at highest risk, hence, is crucial to optimize patient triage, particularly with respect to ICU capacities.

COVID-19 is considered primarily a respiratory disease; however, patients with cardiovascular disease represent a vulnerable population during this pandemic. Furthermore, cardiac injury in patients with COVID-19, defined as a rise of highly sensitive troponin levels, has been identified as a strong predictor of morbidity and mortality in COVID-19 patients. The prevalence of cardiac injury in hospitalized patients was reported to range between $20 \%$ and $30 \%[5,6]$. The specific causes of troponin rise in COVID-19 in patients without cardiac conditions, such as acute coronary syndrome (ACS), aortic stenosis, hypertrophic cardiomyopathy, and tachycardia

\footnotetext{
A. A. Kammerlander, MD, $\mathrm{PhD}$.

ao. Univ. Prof. Dr. J. Mascherbauer $(\bowtie)$

Division of Cardiology, Department of Medicine II, Medical

University of Vienna, Waehringer Guertel 18-20, 1090 Wien, Austria

julia.mascherbauer@meduniwien.ac.at
}

arrhythmia are incompletely understood but two main theories prevail. Firstly, elevated troponin levels are often observed in patients with critical illnesses and have been linked to the systemic inflammatory state (cytokine storm), which can involve damage to any organ, including the heart [7]. Secondly, viral diseases are known to potentially cause myocardial inflammation/myocarditis [8]. Indeed, several cases of fulminant myocarditis in patients with COVID19 have been reported [9]; however, comprehensive clinical and imaging work-up of these patients in prospective cohorts is lacking.

Frequently, cardiologists face questions by patients as well as other healthcare professionals about cardiovascular-specific aspects of COVID-19. The intention of this editorial is to provide useful sources with up to date information and discuss some of the most frequently asked questions.

(1) Should I discontinue ACE inhibitor/ARB inhibitors in patients with COVID-19? The SARS-CoV-2 enters human cells by binding to the angiotensin-converting enzyme 2 (ACE2) receptor. Concerns exist that ACE inhibitors (ACE-I) and angiotensin receptor blockers (ARB) may be harmful due to an upregulation of ACE2, which could facilitate infections with SARS$\mathrm{CoV}-2$ or increase disease severity [10]. Given the frequent use of ACE-I and ARB in patients suffering from hypertension, coronary artery disease, heart failure, and chronic kidney disease, such considerations lead to great uncertainty among patients and physicians. In addition, early observational data reported that patients taking ACE-I and ARB were at higher risk for a dismal outcome in COVID-19 [11]; however, these early reports did not take into account the higher risk profile which we expect in patients taking ACE-I and $\mathrm{ARB}$ as compared to those having no previous medication. Conversely, some animal models sug- 
gested a protective effect of ACE-I and ARB in acute lung injury [12, 13]. Several retrospective studies reported that patients with COVID-19 taking ACE-I or ARB were at lower risk for mortality compared to patients without ACE-I or ARB in adjusted models $[14,15]$. Dozens of prospective trials randomizing patients with COVID-19 to either ACE-I and ARB or placebo are currently ongoing, which will augment our knowledge on potential benefits and harms of ACE-I and ARB in COVID-19.

As of June 2020, all major cardiovascular scientific organizations and official health regulatory bodies recommend not to discontinue ACE-I and ARB in patients with COVID-19 [16, 17]. A beneficial effect, however, remains uncertain and ACE-I and ARB should not be used as treatment for COVID-19 outside of the setting of clinical trials [18].

Updated information may be found under: https:// www.covid19treatmentguidelines.nih.gov/concomita nt-medications/.

(2) My patient with COVID-19 has elevated troponin levels-What now? Cardiac injury, defined as elevated troponin levels, is frequently observed in patients with COVID-19. Potential mechanisms include type I and type II myocardial infarction (MI), Takotsubo syndrome, shock as part of COVID-19, myocarditis with direct viral and cytopathic effects, and cytokine release syndrome [19]. The European Association of Percutaneous Cardiovascular Interventions (EAPCI) issued a position statement on invasive management in patients with ACS during the COVID-19 pandemic [19]. The EAPCI recommends that in cases of mild troponin elevation $(<2-3$ times the upper limit of normal), particularly in older patients with pre-existing cardiac conditions, a work-up for type $1 \mathrm{MI}$ is not indicated, unless strongly indicated by clinical presentation and electrocardiograph (ECG) findings. Marked elevations in cardiac troponin levels (e.g. $>5$ times the upper limit of normal) may be found in acute respiratory syndrome, tachycardia, systemic hypoxemia, shock as part of COVID-19, myocarditis, Takotsubo syndrome, or type 1 MI triggered by COVID-19. If symptoms and ECG findings are not suggestive of type $1 \mathrm{MI}$, the EAPCI recommends that echocardiographic work-up should be considered in order to help diagnose the underlying cause [19].

Percutaneous coronary intervention (PCI) centers have incorporated COVID-19-specific triage systems, in accordance with local regulations and advisory statements. Concerns exist that patients with ACS may not attend medical services due to fear of an infection with SARS-Cov-2. Indeed, studies demonstrated a $40 \%$ drop in ST-elevation myocardial infarction (STEMI) activations in PCI centers [20]. Patients and healthcare professionals should be encouraged to not delay consultation of PCI centers if symptoms are suggestive of ACS.
Of note, several antiviral drugs investigated for treatment of COVID-19 are known to potentially cause myocardial damage but limited data on the clinical significance are available at this time [21].

Updated information may be found under: https:// www.escardio.org/Education/COVID-19-and-Cardiol ogy.

(3) Should I use anticoagulation in patients with COVID-19? Current literature suggests that patients with COVID-19 are at increased risk for venous thromboembolism (VTE). In critically ill patients, VTE is frequent but seems to occur much more frequently in patients with COVID-19, with reported incidence rates of up to one third in ICU patients [22]. The US National Institutes of Health $(\mathrm{NIH})$ provide regularly updated recommendations on anticoagulation strategies in patients with COVID-19 [21].

In nonhospitalized patients there are no data to support any use of anticoagulation. Patients receiving antiplatelet or anticoagulant treatment for underlying conditions should continue their medications irrespective of a COVID-19 diagnosis [21].

In patients hospitalized with COVID-19, both NIH and the anticoagulation forum recommend pharmacologic VTE prophylaxis [21, 23]; however, irrespective of laboratory findings, there are currently insufficient data to support routine screening for VTE in patients without clinical signs suggestive of VTE. Patients with confirmed thromboembolic events who have been diagnosed with COVID-19 should receive standard of care anticoagulation treatment [21].

Updated information may be found under: https:// www.covid19treatmentguidelines.nih.gov/antithromb otic-therapy/.

(4) How long can invasive procedures for cardiac conditions be postponed? As healthcare systems around the globe experience unprecedented challenges, clinical management of all chronic cardiac conditions has changed dramatically. The European Society of Cardiology (ESC) issued a guidance document on how to prioritize management in patients with cardiac conditions [24]. Patients with ACS, left main PCI, battery replacement in case of battery end of life and pacing dependency, and valvular heart disease, who are hemodynamically unstable should be considered emergency and urgent priority and invasive procedures should not be postponed.

Symptomatic patients with severe aortic stenosis should undergo valve replacement within 3 months in cases of advanced disease state (aortic valve area $<0.6 \mathrm{~cm}^{2}$, mean transvalvular gradient $>60 \mathrm{~mm} \mathrm{Hg}$ ). All cases should be discussed by the interdisciplinary heart team and indications for transcatheter aortic valve implantation (TAVI) extended to intermediate and selected low-risk patients. Increased use of transfemoral TAVI (when feasible) may allow optimal utilization of resources by avoiding general anesthesia 
and intubation, shortening (or preventing) ICU stay and accelerating hospital discharge and recovery.

Updated information may be found under: https:// www.escardio.org/Education/COVID-19-and-Cardiol ogy/ESC-COVID-19-Guidance.

Conflict of interest A.A. Kammerlander and J. Mascherbauer declare that they have no competing interests.

\section{References}

1. Zhu N, Zhang D, Wang W, et al. A Novel Coronavirus from Patients with Pneumonia in China, 2019. N Engl J Med. 2020;382(8):727-33.

2. Guan WJ, Ni ZY, Hu Y, et al. Clinical Characteristics of Coronavirus Disease 2019 in China. N Engl J Med. 2020;382(18):1708-1720. https://doi.org/10.1056/ NEJMoa2002032

3. Munster VJ, Koopmans M, van Doremalen N, van Riel D, de Wit E. A novel Coronavirus emerging in China-key questions for impact assessment. $\mathrm{N}$ Engl J Med. 2020;382(8):692-4.

4. Chen N, Zhou M, Dong X, et al. Epidemiological and clinical characteristics of 99 cases of 2019 novel coronavirus pneumonia in Wuhan, China: a descriptive study. Lancet. 2020;395(10223):507-13.

5. Shi S, Qin M, Shen B, et al. Association of Cardiac Injury With Mortality in Hospitalized Patients With COVID-19 in Wuhan, China. Jama Cardiol. 2020; https://doi.org/10. 1001/jamacardio.2020.0950.

6. Guo T, Fan Y, Chen M, et al. Cardiovascular implications of fatal outcomes of patients with Coronavirus disease 2019 (COVID-19). JAMA Cardiol. 2020; https://doi.org/10.1001/ jamacardio.2020.1017.

7. Guest TM, Ramanathan AV, Tuteur PG, Schechtman KB, Ladenson JH, Jaffe AS. Myocardial injury in critically ill patients. A frequently unrecognized complication. JAMA. 1995;273(24):1945-9.

8. Fung G, Luo H, Qiu Y, Yang D, McManus B. Myocarditis. Circ Res. 2016;118(3):496-514.

9. Hu H, Ma F, WeiX, Fang Y. Coronavirus fulminant myocarditis saved with glucocorticoid and human immunoglobulin. Eur Heart J. 2020; https:// doi.org/10.1093/eurheartj/ ehaal90.

10. Wan Y, Shang J, Graham R, Baric RS, Li F. Receptor recognition by the novel Coronavirus from Wuhan: an analysis based on decade-long structural studies of SARS Coronavirus. J Virol. 2020; https://doi.org/10.1128/jvi.0012720.

11. Novel Coronavirus Pneumonia Emergency Response Epidemiology Team. Vital surveillances: the epidemiological characteristics of an outbreak of 2019 novel coronavirus diseases (COVID-19) —China, 2020. China CDC Weekly. 2020. http://weekly.chinacdc.cn/en/article/id/e53946e2c6c4-4le9-9a9b-fea8dbla8f51. Accessed 7 May 2020.

12. Yang $P, \mathrm{Gu} \mathrm{H}, \mathrm{Zhao} \mathrm{Z,} \mathrm{et} \mathrm{al.} \mathrm{Angiotensin-converting} \mathrm{enzyme}$ 2 (ACE2) mediates influenza H7N9 virus-induced acute lung injury. Sci Rep. 2014;4:7027.
13. Mackey K, King VJ, Gurley S, et al. Risks and impact of Angiotensin-converting enzyme inhibitors or Angiotensinreceptor blockers on SARS-coV-2 infection in adults. Ann Intern Med. 2020; https://doi.org/10.7326/m20-1515.

14. Li J, WangX, Chen J, Zhang H, DengA. Association of ReninAngiotensin system inhibitors with severity or risk of death in patients with hypertension hospitalized for Coronavirus disease 2019 (COVID-19) infection in Wuhan, China. Jama Cardiol. 2020; https://doi.org/10.1001/jamacardio.2020. 1624.

15. Zhang P, Zhu L, Cai J, et al. Association of inpatient use of Angiotensin converting enzyme inhibitors and Angiotensin II receptor blockers with mortality among patients with hypertension hospitalized with COVID-19. Circ Res. 2020;126(12):1671-1681. https://doi.org/10.1161/ CIRCRESAHA. 120.317134

16. EMA, editor. EMA advises continued use of medicines for hypertension, heart or kidney disease during COVID19 pandemic. 2020. https://www.ema.europa.eu/en/ documents/press-release/ema-advises-continued-usemedicines-hypertension-heart-kidney-disease-duringcovid-19-pandemic_en.pdf. Accessed 24 May 2020.

17. HFSA/ACC/AHA Statement Addresses Concerns Re: Using RAAS Antagonists in COVID-19. https://www. acc.org/latest-in-cardiology/articles/2020/03/17/08/59/ hfsa-acc-aha-statement-addresses-concerns-re-usingraas-antagonists-in-covid-19 (accessed on May 24, 2020).

18. Considerations for Certain Concomitant Medications in Patients with COVID-19. NIH COVID-19 Treatment Guidelines (https://www.covid19treatmentguidelines.nih.gov/ concomitant-medications/ accessed on May 24,2020).

19. Chieffo A, Stefanini GG, Price S, et al. EAPCI position statement on invasive management of acute coronary syndromes during the COVID-19 pandemic. Eur Heart J. 2020;41(19):1839-51.

20. Garcia S, Albaghdadi MS, Meraj PM, et al. Reduction in STsegment elevation cardiac catheterization laboratory activations in the United States during COVID-19 pandemic. J Am Coll Cardiol. 2020; https://doi.org/10.1016/j.jacc. 2020.04.011.

21. Antithrombotic Therapy in Patients with COVID-19. NIH COVID-19 Treatment Guidelines. (https://www. covid19treatmentguidelines.nih.gov/antithrombotic-ther apy/ accessed on May 24, 2020).

22. Klok FA, Kruip M, van der Meer NJM, et al. Incidence of thrombotic complications in critically ill ICU patients with COVID-19. Thromb Res. 2020; https://doi.org/10.1016/j. thromres.2020.04.041.

23. Barnes GD, Burnett A, Allen A, et al. Thromboembolism and anticoagulant therapy during the COVID-19 pandemic: interim clinical guidance from the anticoagulation forum. J Thromb Thrombolysis. 2020; https://doi.org/10.1007/ s11239-020-02138-z.

24. ESC Guidance for the Diagnosis and Management of CV Disease during the COVID-19 Pandemic. https://www. escardio.org/Education/COVID-19-and-Cardiology/ESCCOVID-19-Guidance (accessed on May 25, 2020).

Publisher's Note Springer Nature remains neutral with regard to jurisdictional claims in published maps and institutional affiliations. 